Thomas Reske*, Katharina Wulf, Sabine Illner, Thomas Eickner, Niels Grabow, KlausPeter Schmitz, Stefan Siewert, Valeria Khaimov

\title{
Non-destructive analysis of drug distribution in nonwovens as drug-delivery systems for biomedical applications
}

\begin{abstract}
Analysis of the active ingredient distribution of medical devices is typically performed using Raman spectroscopy, a method that is fast and inexpensive [1]. In addition, it offers the advantage of non-destructive analysis without the need for special sample preparation. Assuming that all components are Raman-active and present in sufficient quantities, their distribution can be well represented.
\end{abstract}

The drug distribution in dexamethasone-loaded polymer nonwovens was investigated in order to draw conclusions on the quality of the fleece batches and to make predictions for the release behavior.

In the present study, dexamethasone (DMS), a glucocorticoid was used as the active ingredient. Qualitative and quantitative studies of the content of DMS in polymer films by means of Raman spectroscopy have already been carried out in the working group [2].

A representative square section was examined to describe the distribution of active ingredients. The required number of measurement points (spectra) was determined earlier [2].

Keywords: Raman spectroscopy, dexamethasone distribution, polymeric nonwovens, Poly-L-Lacide, Polydioxanone.

https://doi.org/10.1515/cdbme-2021-2128

\footnotetext{
*Corresponding author: Thomas Reske: Institute for Implant Technology and Biomaterials e.V., Friedrich-Barnewitz-Str. 4, 18119 Rostock-Warnemünde, Germany, e-mail: thomas.reske@uni-rostock.de Katharina Wulf, Sabine IIIner, Thomas Eickner, Niels Grabow: Institute for Biomedical Engineering, Rostock University Medical Center, Rostock-Warnemünde, Germany

Klaus-Peter Schmitz, Stefan Siewert: Institute for ImplantTechnology and Biomaterials e.V., Rostock-Warnemünde, Germany

Valeria Khaimov: Institute for ImplantTechnology and Biomaterials e.V., Friedrich-Barnewitz-Strasse 4, 18119 RostockWarnemünde, Germany
}

\section{Introduction}

In medical technology, drug-loaded nonwovens can be used in a variety of ways. This requires that their production, which is preceded by an electrospinning process, can be carried out with repeatable quality. Furthermore, it is important that the drug is uniformly distributed to obtain a controllable drug release.

Raman spectroscopy is suitable for visualizing the drug distribution in a non-destructive manner without the need for extensive sample preparation. The investigations were carried out here for the first time on samples of polyester-based nonwovens such as poly-L-lactide (PLLA) and polydioxanone (PDO) loaded with dexamethasone (DMS) in a similar manner as described previously [2].

The degradation behavior of these polymers, which also has an influence on the release behavior, has already been reported [3]

\section{Materials and methods}

\subsection{Samples}

Drug-containing nonwovens with a thickness of ca. $100 \pm 50 \mu \mathrm{m}$ and with a DMS content of $20 \%$ were produced by an electrospinning process. Materials used were PLLA (Resomer L210, Evonik), PDO (Resomer X206 S, Evonik) and a blend of both materials with a PLLA/PDO ratio of $30 / 70 \%(w / w)$. Three samples from each nonwoven batch were analyzed.

\subsection{Overview image}

All measurements were performed using a confocal Raman microscope Alpha300R (WITec GmbH, Ulm). Overview images of all samples with an area of $2.000 \mu \mathrm{m} \times 2.000 \mu \mathrm{m}$ were recorded by means of a Zeiss EC "Epiplan-Neofluar" 
10x objective (NA 0.25 ). Therefore 25 single images were stitched.

\subsection{Analysis via area scan measurement}

Raman measurements were performed within an area of $500 \mu \mathrm{m} \times 500 \mu \mathrm{m}$ ("area scan") with $25 \times 25$ measuring points. An integration time of $2 \mathrm{~s}$ per measuring point was used. A Raman spectrum was recorded at each measurement point. The visualization of the measurement points was conducted via a heat map in which the intensity of a certain band is displayed. These bands are 1,670 rel. $\mathrm{cm}^{-1}$ for DMS, 1,780 rel. $\mathrm{cm}^{-1}$ for PLLA and 1,745 rel. $\mathrm{cm}^{-1}$ for PDO. High intensities are shown with yellow color and low intensities with black color.

An output power of a Nd:YAG $532 \mathrm{~nm}$ laser of $10 \mathrm{~mW}$ was used. Measurements were also performed using a Zeiss EC "Epiplan-Neofluar" 10x objective (NA 0.25) (Carl Zeiss AG, Oberkochen, Germany).

\section{Results and discussion}

Fig. 1 shows an exemplary overview image with such an investigated area of PLLA sample no. 1.

The PLLA and PDO nonwovens generally showed a homogeneous surface structure. However, slight wrinkles occurred during storage, which can also be observed in Fig. 1.

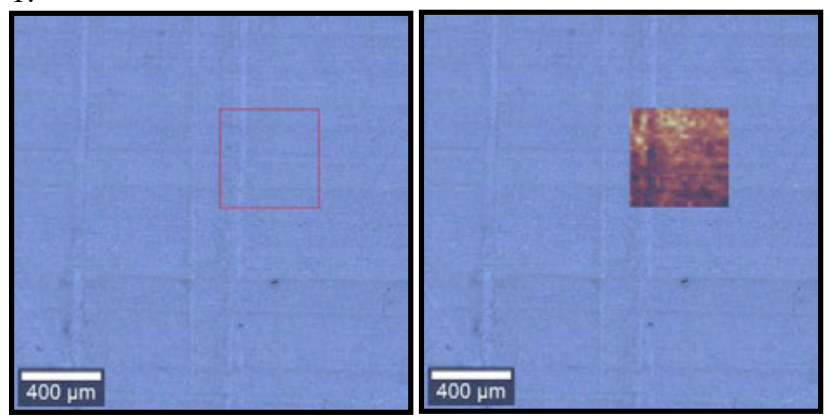

Figure 1: Exemplary overview image of PLLA/DMS-nonwoven. The red square shows the area where Raman measurements were performed. On the right side the overlay image with the area scan is shown.

Two vertical stripes are visible on the left side of both area scans of PLLA/DMS (Figure 2). These are due to the abovementioned unevenness in the z-plane. This is more clearly visible in the overlay image.
Furthermore, it can be seen in the area scans that DMS and polymer signals show equal intensities at the same positions (Figure 2). This was observed in PLLA/DMS and PDO/DMS samples and is due to the fact that the investigated nonwovens were not completely focused within the z-plane in the analyzed area. Thus, considering the structure of the nonwovens, it can be stated that the DMS distribution is homogeneous within one sample.

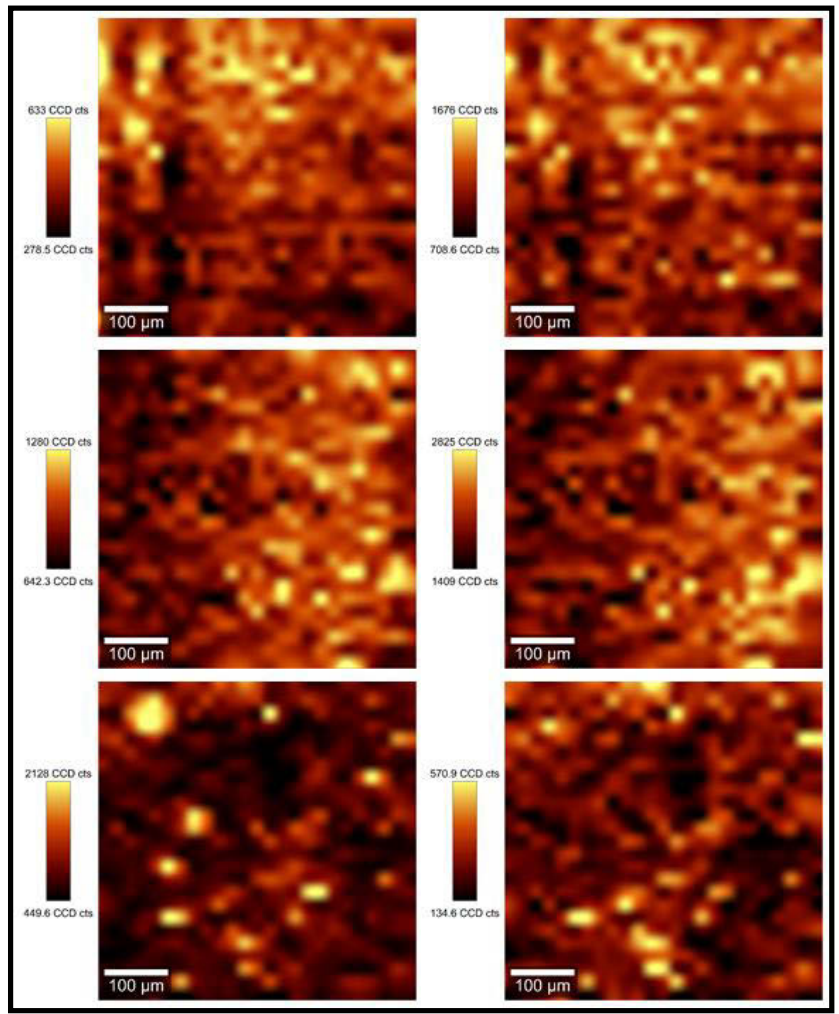

Figure 2: Large Area Scan for DMS (left side) and polymer (right side). In the top line PLLA/DMS is shown, in the middle PDO/DMS and below the blend (PLLA/PDO)/DMS. There are clear differences in the blend; in the upper left corner, for example, DMS is concentrated in high intensity in a larger point-like area.

When looking at the blend, it is noticeable that the active ingredient and polymer bands are not always present in the same intensity. This was not necessarily to be expected for a three-component system, but it does have consequences for the drug release.

To evaluate the DMS distribution within a nonwoven batch, area scans were performed on three samples each. Of the 625 Raman spectra measured, an average spectrum was determined. 


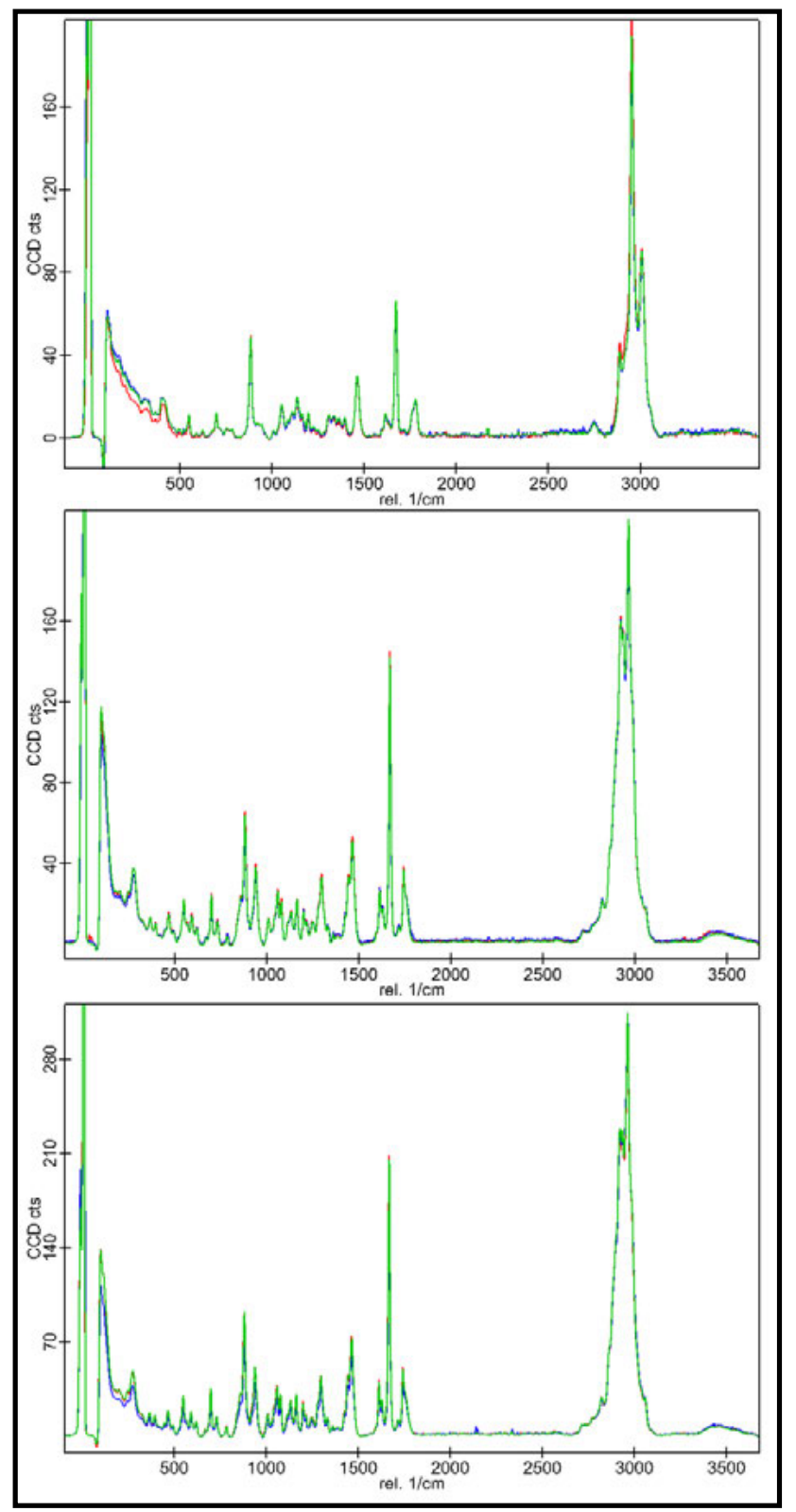

Figure 3: Averaged Raman spectra ( $n=3$, each). In the top line PLLA/DMS is shown, in the middle PDO/DMS and below the blend (PLLA/PDO)/DMS. The x-axis shows the wavenumber and the $y$-axis the intensity.

The comparison of the three averaged spectra per material shows that the spectra are almost congruent with each other and thus there is very good reproducibility within a batch (Figure 3).
The spectra of PDO/DMS and the blend (PLLA/PDO)/DMS are very similar. Due to the lower content of PLLA in the blend, PLLA bands are overlaid completely by PDO in the blend spectrum.

\section{Conclusion}

The Raman analysis is performed non-destructively with a laser output power of $10 \mathrm{~mW}$ and 625 measuring points. The selected parameters allow subsequent release studies of single samples.

The nearly congruent averaged spectra of the area scans for one material show that the preparation of the samples of one batch was done with a high degree of reproducibility. The active ingredient is homogeneously distributed in PLLA/DMS and PDO/DMS.

The results can be used to make predictions on the course of a release curve. For example, initial values from a release study currently in progress show that, in the case of the blend samples, there is a greater standard deviation than in the case of DMS release from the pure polymers for the same number of samples. This is consistent with expectations for a less homogeneous sample.

\section{Author Statement}

Research funding: Partial financial support by the European Regional Development Fund (ERDF) and by the Federal Ministry of Education and Research (BMBF) within RESPONSE "Partnership for Innovation in Implant Technology" is gratefully acknowledged.

Conflict of interest: Authors state no conflict of interest.

\section{References}

[1] Belu A, Mahoney C, Wormuth K. Chemical imaging of drug eluting coatings: Combining surface analysis and confocal Raman microscopy. J Control Release 2008; 126:111-121.

[2] Reske T, Wulf K, Eickner T, Grabow N, Schmitz KP, Siewert S. Non-destructive analysis of drug content in polymer coatings with Raman spectroscopy. Curr. Dir. Biomed. Eng. 2019; 5:469-472.

[3] Reske T, Eickner T, Grabow N, Schmitz KP, Siewert S. Accelerated Degradation of polymeric surgical suture materials. Curr. Dir. Biomed. Eng. 2020; 6:458-460. 